\title{
PROCESSO DE DESCARBURAÇÃO VIA VOD DE AÇOS INOXIDÁVEIS COM ALTO TEOR DE CARBONO INICIAL*
}

\author{
Robson Leandro Silva ${ }^{1}$ \\ Marcelo Breda Mourão ${ }^{2}$ \\ Egberto Antônio Possente ${ }^{3}$ \\ Mario Cesar Mantovani ${ }^{4}$ \\ Luiz Roberto Moraes Junior ${ }^{5}$ \\ Bruno Pessoa Ramos ${ }^{6}$
}

\section{Resumo}

O processo de descarburação de aço inoxidável contendo alto teor de carbono inicial via processo VOD foi estudado utilizando o aço AISI 304. Devido a questões operacionais, o carbono inicial aplicado no processo VOD da Villares Metals é próximo a $1 \%$ e a pressão de trabalho de 600 mbar. $O$ objetivo do trabalho foi encontrar o instante em que se torna possível a redução da pressão do sistema de acordo com o carbono inicial do processo. Para isso foram realizados testes em corridas industriais de $25 \mathrm{t}$ interrompendo o sopro de oxigênio e realizando análise química em diferentes tempos de sopro. O resultado dos testes mostrou que após 11 min de sopro de oxigênio é possível reduzir a pressão do sistema levando em consideração as limitações operacionais, podendo assim reduzir a oxidação de elementos de liga durante essa etapa.

Palavras-chave: VOD; Descarburação; Aço inoxidável; Cinética.

\section{DECARBURIZATION OF STAINLESS STEEL VIA VOD WITH HIGH INITIAL CARBON CONTENT}

\section{Abstract}

The decarburization process of stainless steel with high initial carbon content by VOD process was studied using the AISI 304. Due to operational issues of Villares Metals S.A. the initial carbon content of VOD process is close to $1 \%$ and the total pressure is $600 \mathrm{mbar}$. The objective of this work was to find the exact moment which it becomes possible to reduce the system pressure according to the initial carbon. The industrial trials were carried out by interrupting the oxygen blowing in three different periods of time. Steel samples were taken in each period of time for chemical analysis together temperature measurements. The results showed that after $11 \mathrm{~min}$ of oxygen blowing is possible to reduce the system pressure take into account the operational limitation, becoming it possible to reduce the alloys oxidation during this step.

Keywords: VOD; Decarburization; Stainless Steel; Kinetic.

1 Engenheiro Metalurgista, Engenheiro de Processos, Dep. de Engenharia de Processos, Villares Metals S.A., Sumaré, SP.

2 Membro da ABM, Doutor em Engenharia Metalúrgica, Professor Doutor, Dep. de Engenharia Metalúrgica, Universidade de São Paulo, São Paulo, SP.

3 Especialista em Processos, Aciaria, Villares Metals S.A., Sumaré, SP.

4 Membro da ABM, Doutor em Engenharia Metalúrgica, Pesquisador, Dep. de Pesquisa e Desenvolvimento, Villares Metals S.A., Sumaré, SP.

5 Engenheiro de Materiais, Gerente da Aciaria Convencional, Aciaria, Villares Metals S.A., Sumaré, SP.

6 Engenheiro Metalurgista, Diretor da Aciaria, Aciaria, Villares Metals S.A., Sumaré, SP. 


\section{INTRODUÇÃO}

O crescimento da produção de aço inoxidável no mundo refletiu a importância destes aços na engenharia. Em 1980 a produção mundial de aço inoxidável foi de 6,9 milhões de toneladas, aumentando para 41,5 milhões em 2015, crescimento de $5,27 \%$ ao ano. Entre 1980 e 2015, este crescimento da produção foi o maior crescimento considerando os principais metais usados na indústria, como por exemplo, alumínio $(3,86 \%)$ e o cobre $(2,74 \%)$, segundo e terceiro lugares respectivamente [1]. A produção brasileira de aço inoxidável em 2015 foi de 401 mil toneladas, estando em $13^{\circ}$ lugar, sendo a China a maior produtora com 21,6 milhões, 52\% da produção mundial [1] [2].

\section{FUNDAMENTOS DA DESCARBURAÇÃO NO VOD}

A etapa principal do processo de fabricação dos aços inoxidáveis é a descarburação, sendo que os dois principais processos utilizados são: o VOD (Vacuum Oxygen Decarburization) no qual a pressão parcial do $\mathrm{CO}_{(\mathrm{g})}$ é reduzida mediante a aplicação de vácuo, já no processo AOD (Argon Oxygen Decarburization) a pressão parcial de $\mathrm{CO}_{(\mathrm{g})}$ é reduzida (diluição) pela injeção de argônio e/ou nitrogênio [3][4]. As principais etapas do processo são divididas em três, considerando o processo VOD: Sopro de oxigênio, descarburação e redução, sendo a sequência do processo esquemático mostrado na Figura 1 [5][6][7].

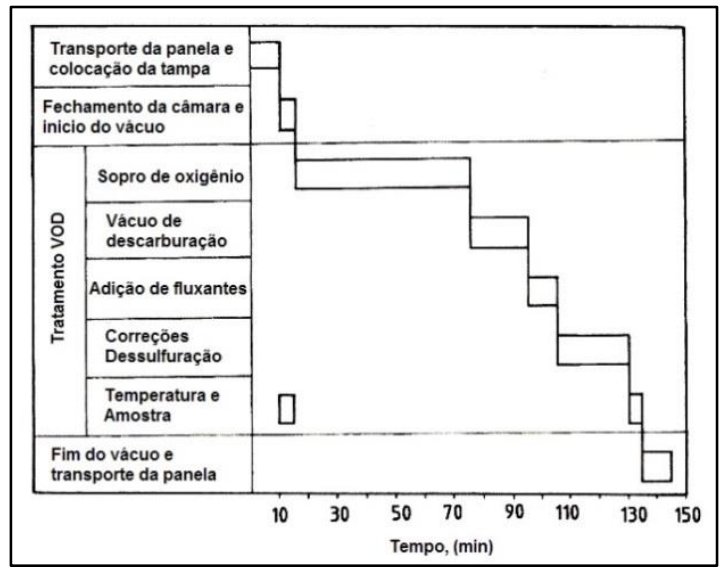

Figura 1. Sequência do processo VOD [7].

A taxa de descarburação pode ser controlada pelo ajuste das variáveis: vazão de oxigênio, distância lança-banho, vazão de sucção e vazão de gás inerte. Modernas práticas de VOD incluem um programa de sopro calculado em tempo real para cada corrida [8]. Como exemplo, segundo Teeuwsen [8], o programa de sopro para descarburar um aço inoxidável em uma determinada planta foi desenvolvido ajustando-se a vazão de oxigênio, pressão de vácuo e a distância da lança ao banho. Atualmente existem diversas práticas para ajustar o processo de descarburação, específicas à cada planta de modo a se obter um menor tempo de processo e menor quantidade de redutor utilizado na etapa de redução. A Figura 2 mostra um perfil de sopro de oxigênio para uma corrida de aço inoxidável de 50 ton, segundo Teeuwsen [8]. 


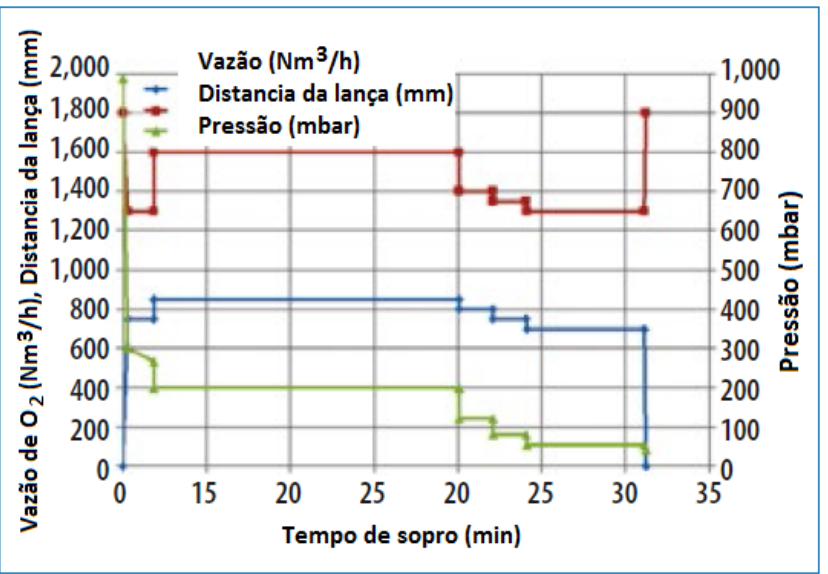

Figura 2. Programa de sopro de um aço inoxidável [8].

A principal reação química que ocorre durante o processo de descarburação está descrita na Equação (1), sendo a constante de equilíbrio dada pela Equação (2).

$$
\begin{aligned}
<\mathrm{Cr}_{2} \mathrm{O}_{3}>+3 \underline{C}=2 \underline{C r}+3(\mathrm{CO}) \quad \Delta \mathrm{G}^{0}= & 758.857-481,27 \cdot T \quad(\mathrm{~J}) \\
& \frac{P_{C O}^{3} \cdot a_{C r}^{2}}{a_{C}^{3} \cdot a_{C_{2} O_{3}}}=\exp \left(\frac{-\Delta G^{0}}{R \cdot T}\right)
\end{aligned}
$$

Como mostra a Figura 3, a reação de descarburação (1) é favorecida com a redução da pressão do sistema, podendo ocorrer em temperaturas menores, reduzindo assim a oxidação do cromo e minimizando o desgaste do refratário da panela [3][9].

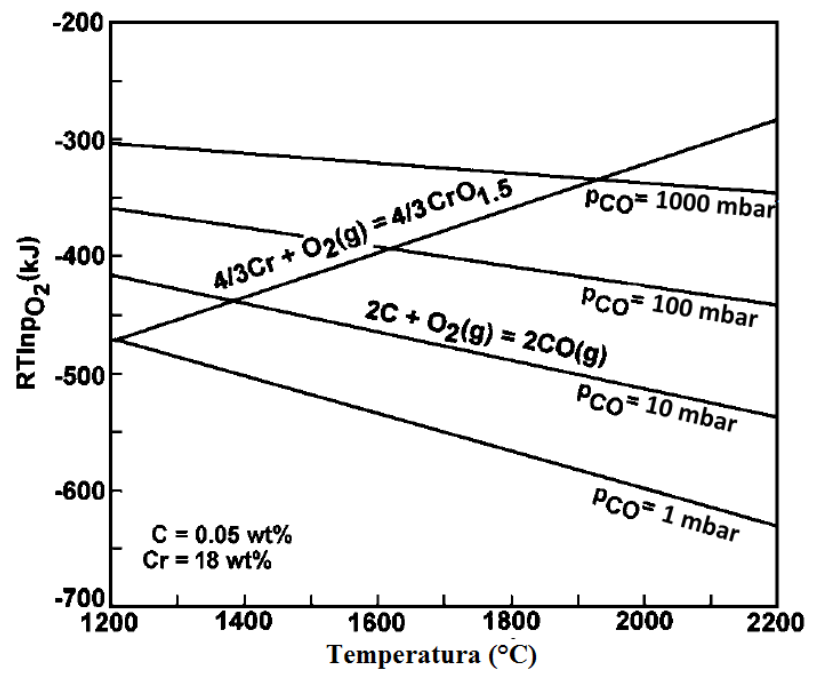

Figura 3. Diagrama de Ellingham para a reação de oxidação do cromo e carbono [5].

O teor de carbono crítico é definido como o teor abaixo do qual o cromo se oxidará. Este valor aumenta com a atividade do cromo e com a diminuição da temperatura, conforme mostra a Figura 4 (a) [10]. A Figura 4 (b) mostra os importantes papéis da atividade do óxido de cromo na escória e temperatura, influenciando o teor de carbono de equilíbrio para um dado teor de cromo [11]. Através da Equação (2) e considerando a composição química típica do aço 
inoxidável AISI 304, o teor de carbono em equilíbrio com o cromo a $1600^{\circ} \mathrm{C}$ e 600 mbar de pressão é de $0,35 \%$. Considerando a mesma reação a uma pressão de 400 mbar e 250 mbar, o teor de carbono é reduzido para 0,25\% e 0,17\% respectivamente.
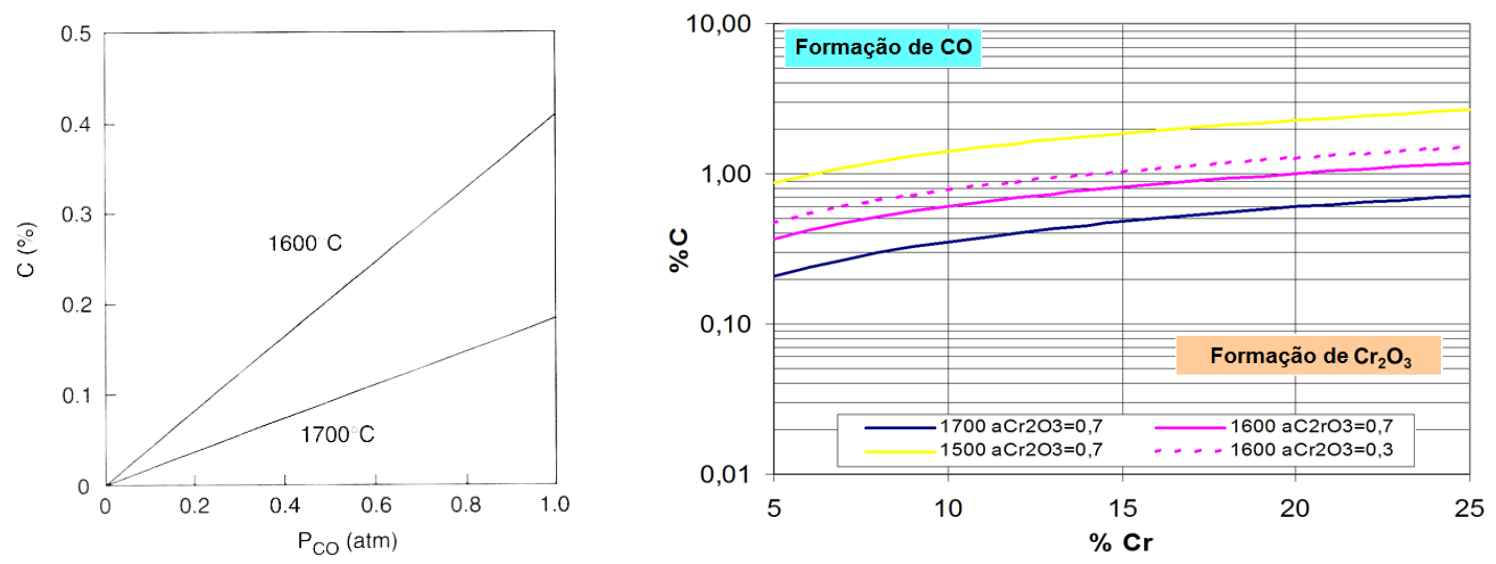

Figura 4. (a) Teor de carbono crítico para um aço com 18\%Cr [10]. (b) Sistema Fe-C-Cr-O a 1 atm [11].

Sabe-se que a fluidodinâmica da panela no processo VOD tem um papel importante na eficiência desse processo. Segundo Krishnakumar [12] a relação ideal entre a profundidade de metal líquido na panela em relação ao seu diâmetro, parâmetro conhecido como "aspect ratio", é de 0,5 a 0,7 para garantir uma borda livre suficiente que evite transbordamentos durante o processo. Segundo Bingel [13] geralmente a borda livre é maior que $1 \mathrm{~m}$. Uma borda livre ampla é necessária, pois a reação de descarburação é muito violenta apesar de esta reação ocorrer próximo à superfície do banho [5]. A pressão ferrostática de uma bolha no aço líquido aumenta 6,7 mbar para cada centímetro de profundidade. Isso que dizer que quanto menor a pressão do sistema, maior será a profundidade onde ocorrerá a descarburação e consequentemente maior o volume de metal líquido envolvido na evolução dos gases. Esta é a razão pela qual a pressão do sistema não poder ser muito baixa durante o sopro de oxigênio [5]. Valores típicos para pressão do sistema durante o sopro são de 100 a 300 mbar segundo a literatura [5][13][14][15]. O carbono desejável para inicio do processo é menor que 1\% já que teores mais altos de carbono podem causar uma "fervura" excessiva, devido ao grande volume de $\mathrm{CO}_{(\mathrm{g})}$ gerado, da mesma forma que ocorre quando se aplica menores pressões durante a etapa de sopro [3]. Valores típicos de carbono inicial ao processo VOD são menores que $0,5 \%$, pois normalmente é aplicado o processo conhecido como tríplex, onde o aço é vazado no conversor AOD após a fusão no Forno Elétrico a Arco (FEA), descarburado (AOD) até atingir teores de carbono menores que $0,5 \%$ e depois continua-se a descarburação no VOD [5][14][15].

No caso do processo de descarburação da Villares Metals, a pressão é mantida constante a 600 mbar exatamente para evitar transbordo já que o "aspect ratio" varia de 0,8 a 1 , valor este mais alto do que é normalmente empregado conforme descrito anteriormente, tal como a borda livre, cujo valor situa-se abaixo dos valores encontrados na maioria das plantas. Este valor de "aspect ratio" maior e a borda livre menor ocorrem devido à geometria e a capacidade atual das panelas da Villares Metals. Além disso, o carbono inicial do processo de descarburação é geralmente mais alto, chegando a teores de $1,10 \%$, já que o aço segue diretamente do FEA para o VOD. 
Segundo a literatura, a taxa de descarburação no inicio do processo é controlada pela transferência de massa de carbono. No início, o oxigênio oxida primeiramente silício e cromo. À medida que as partículas de oxido de cromo ascendem o banho metálico junto com as bolhas de $\mathrm{CO}_{(\mathrm{g})}$, o carbono difunde-se até à superfície e reduz o oxido de cromo de acordo com a Equação 1 [12]. A Equação 3 expressa a taxa de descarburação para esta etapa do processo.

$$
\frac{d \% C}{d t}=-\frac{\rho}{W} \cdot k \cdot A \cdot\left[\% C-\% C_{e}\right]
$$

Onde: $\rho=$ densidade da liga; $\mathrm{W}=$ massa da liga; $\mathrm{k}=$ coeficiente de transferência de massa; $\mathrm{A}$ = área superficial de reação; $\% \mathrm{Ce}_{\mathrm{e}}=$ concentração de carbono de equilíbrio.

Portanto, visando a otimização do processo, é importante conhecer o instante em que o carbono do aço atinge um determinado valor que permita a redução da pressão do sistema, promovendo a continuação da remoção do carbono e reduzindo assim a oxidação dos elementos de liga sem que haja uma evolução muito alta da geração de gases que leve a ocorrência de transbordamentos de metal líquido.

\section{MATERIAIS E MÉTODOS}

Foram utilizadas nas experiências 30 corridas industriais de aço inoxidável AISI 304, produzidas na Aciaria da Villares Metals cuja composição química inicial ao processo VOD e peso nominal estão mostrados na Tabela 1.

Tabela 1. Composição química inicial ao processo VOD do aço inoxidável AISI 304L e o peso nominal da corrida da Villares Metals.

\begin{tabular}{|c|c|c|c|c|c|c|c|}
\hline \multicolumn{7}{|c|}{ Composição química (\%) } & \multirow{2}{*}{$\begin{array}{c}\text { Peso } \\
(t)\end{array}$} \\
\hline C & Si & Mn & $\mathbf{P}$ & $\mathbf{S}$ & $\mathrm{Cr}$ & $\mathrm{Ni}$ & \\
\hline 0,70 & & & & & 17,0 & 6,5 & 25 \\
\hline- & $<0,35$ & $<2,00$ & $<0,045$ & $<0,03$ & - & - & - \\
\hline 1,10 & & & & & 20,0 & 10,0 & 28 \\
\hline
\end{tabular}

A Figura 5 mostra o desenho esquemático do equipamento VOD. As corridas foram produzidas seguindo o fluxo de processo mostrado na Figura 6 . O processo padrão de descarburação do aço AISI V304 e suas principais variáveis de processo estão mostradas na Figura 7. 


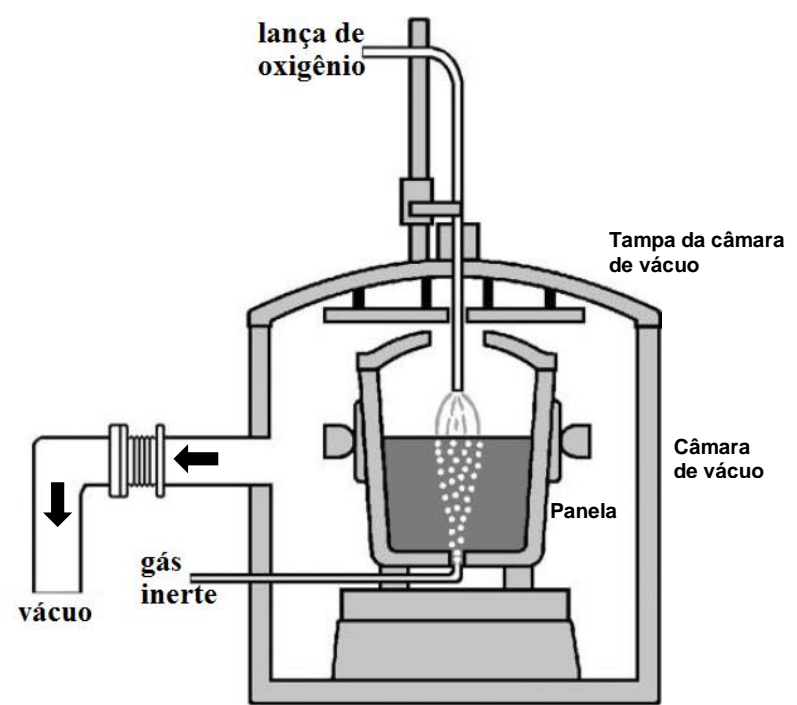

Figura 5. Desenho esquemático do VOD [5].

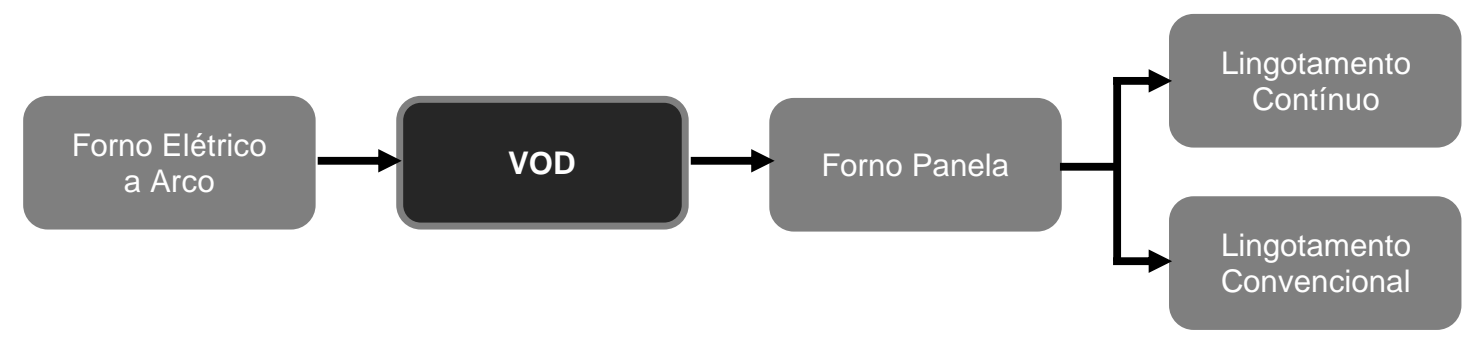

Figura 6. Fluxo de produção do aço AISI 304L na Aciaria da Villares Metals.

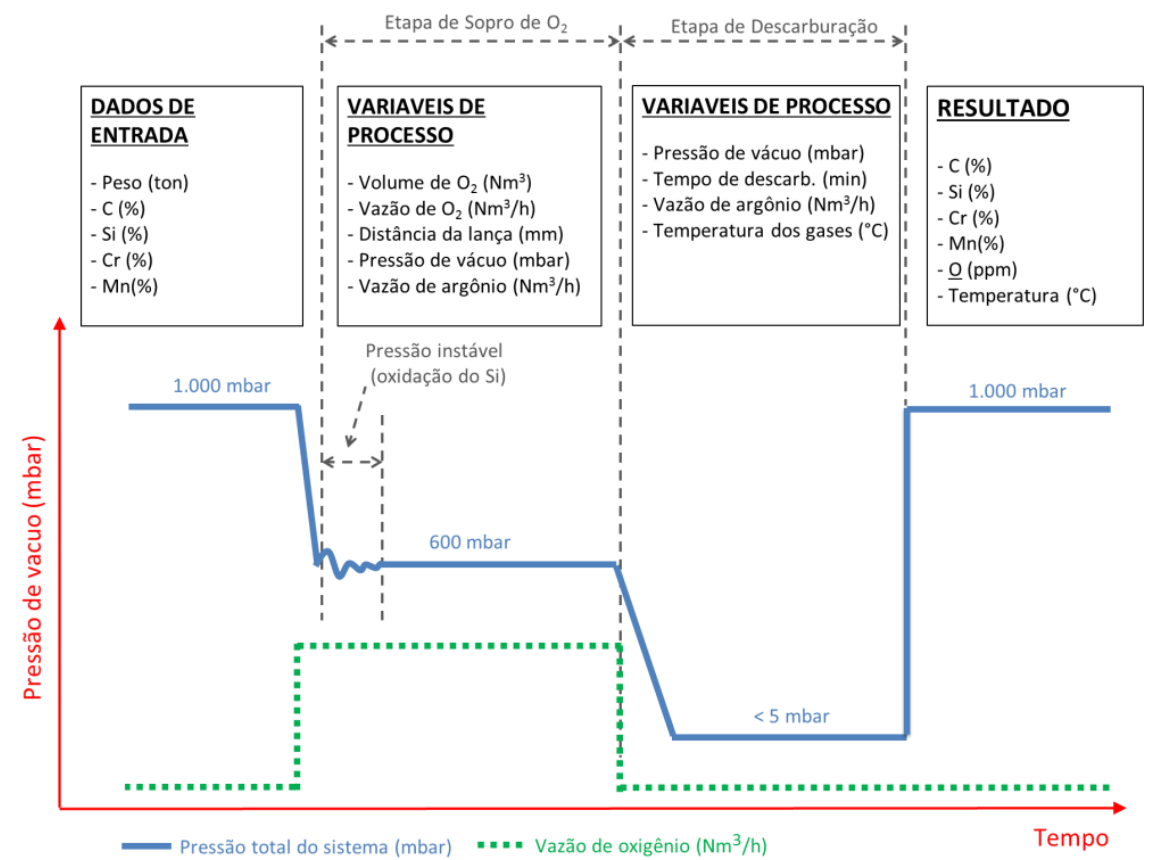

Figura 7. Esquema do processo VOD da Villares Metals para fabricação do aço AISI 304.

Como a pressão total do sistema é regulada para permanecer constante em 600 mbar e nessa condição ocorre preferencialmente a oxidação do silício, o valor da pressão total do sistema oscila em torno dessa pressão devido a variação da rotação do motor de sucção para manter constante essa pressão, pois ainda não há formação de gases dentro da câmara ou a mesma é muito pequena para sobrepor- 
se à pressão de sucção do sistema de vácuo, como mostra a Figura 7, no inicio do processo.

Quando todo o silício é oxidado e consequentemente após o acréscimo de temperatura devido a essa oxidação, inicia-se a oxidação dos elementos de liga, cromo e manganês, mas também do carbono e com isso há geração de gases, principalmente do $\mathrm{CO}_{(\mathrm{g})}$. Nessa condição a pressão do gás gerado compete com a pressão do sistema estabilizando-se no patamar desejado, neste caso em 600 mbar como mostra a Figura 7.

Após a estabilização da pressão, o sopro de oxigênio foi interrompido com 5,8 e 11 min de sopro e coletadas 10 amostras para cada tempo de sopro conforme esquema mostrado na Figura 8. Após essa interrupção, desligou-se a agitação do banho na intenção de parar a reação de descarburação e manteve-se as bombas de sucção do sistema ligadas por um período de tempo de modo a retirar da câmara todo o gás gerado anteriormente e evitar risco de explosão com o ar atmosférico após a abertura da câmara.

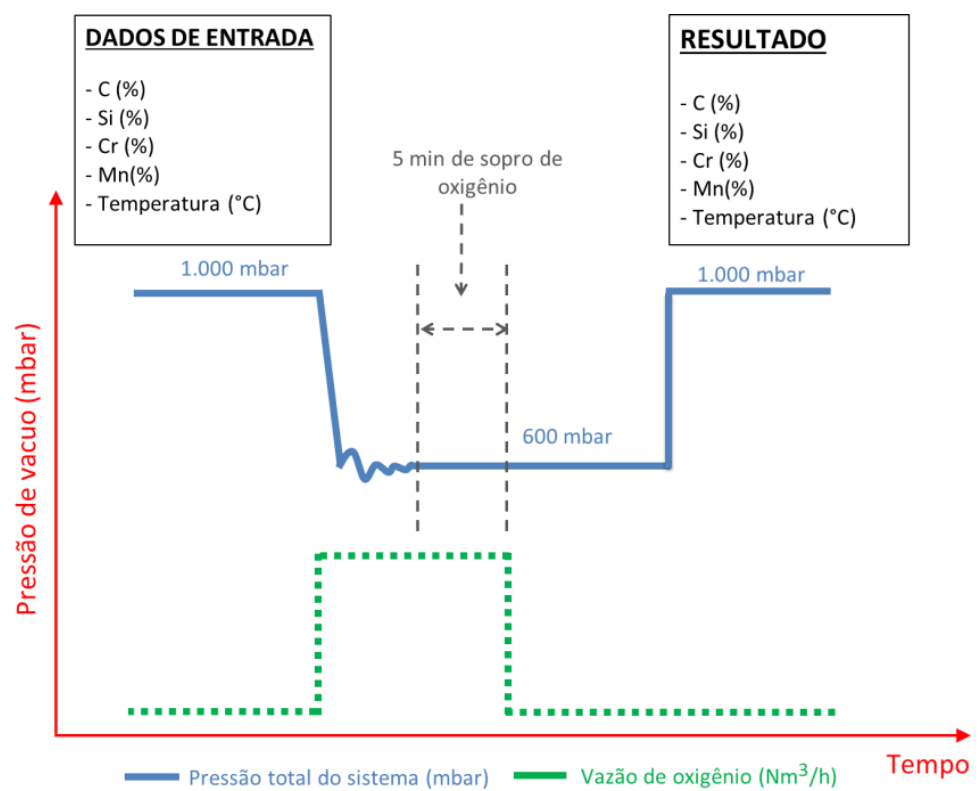

Figura 8: Exemplo do procedimento realizado no teste de interrupção após 5 min de sopro de oxigênio.

As variáveis de processo da etapa de sopro mostradas na Figura 7 foram mantidas constantes, para então serem coletadas amostras para análise de composição química antes e após a etapa de sopro de oxigênio através do método de espectrometria de emissão ótica e de combustão via LECO $^{\circledR}$, realizadas pelo Laboratório de Análise Química da Villares Metals. Foram realizadas medições de temperatura utilizando termopares descartáveis de imersão nas mesmas etapas do processo. De acordo com as análises, foram calculadas a perda de carbono, silício, cromo e manganês e também o acréscimo de temperatura após o processo.

\section{RESULTADOS E DISCUSSÃO}

Em todos os resultados apresentados, quando mencionado "tempo de sopro", este tempo é considerado após a estabilização da pressão total do sistema.

A Figura 9 mostra a perda de carbono em função do tempo de sopro de oxigênio. O carbono inicial das corridas variou entre 0,54 e $1,09 \%$. A perda se mostrou 
proporcional ao tempo de sopro como era esperado, pois maior tempo, maior volume de oxigênio e consequentemente mais carbono oxidado. A perda de carbono juntamente com a Equação (3) foi utilizada para determinar o tempo de sopro em que se torna possível a redução da pressão do sistema mostrado mais adiante. Como explicado anteriormente, no inicio do processo ocorre preferencialmente a oxidação do silício e isso se confirmou como indicado na Figura 10, mostrando que todo o silício foi oxidado independente do seu valor inicial.

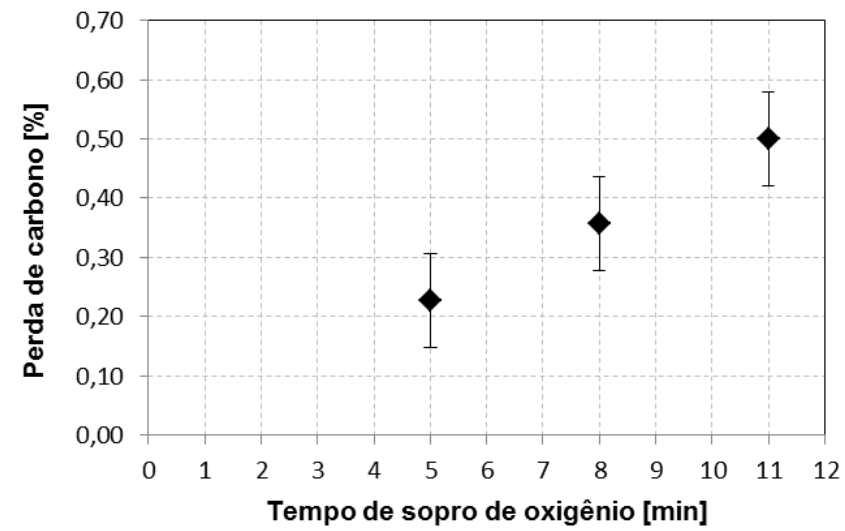

Figura 9: Influência do tempo de sopro na perda de carbono (valor médio de 10 corridas).

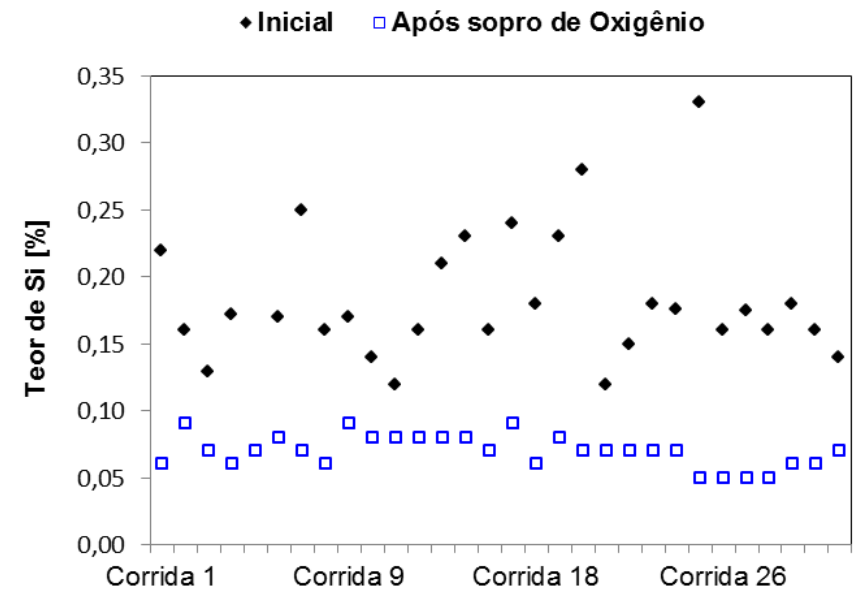

Figura 10: Teor de Si antes e após sopro de oxigênio.

A elevação da temperatura do banho metálico durante o período de amostragem é evidenciada na Figura 11, devido principalmente à oxidação do $\mathrm{Si}$, mas outros elementos de liga tais como Mn e Cr também são oxidados neste período de sopro de oxigênio como mostra a Figura 12. 


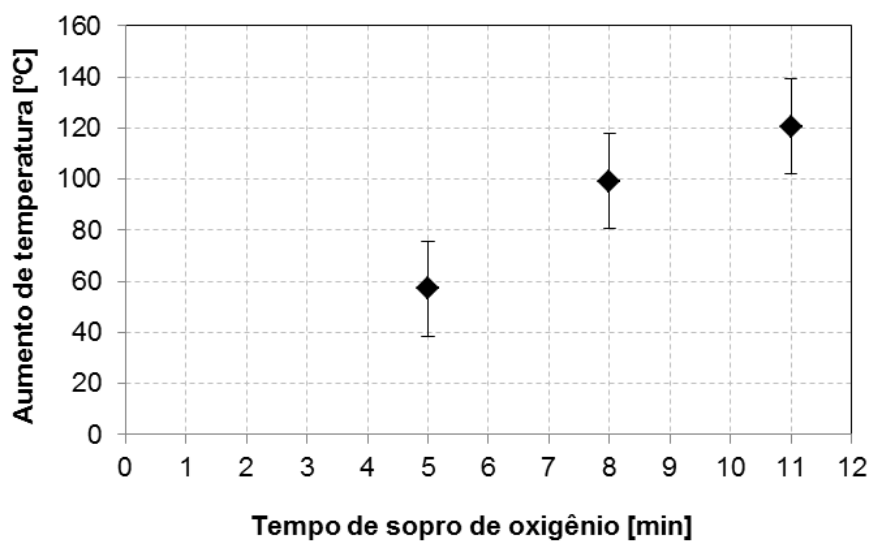

Figura 11: Influência do tempo de sopro no aumento de temperatura (valor médio de 10 corridas).

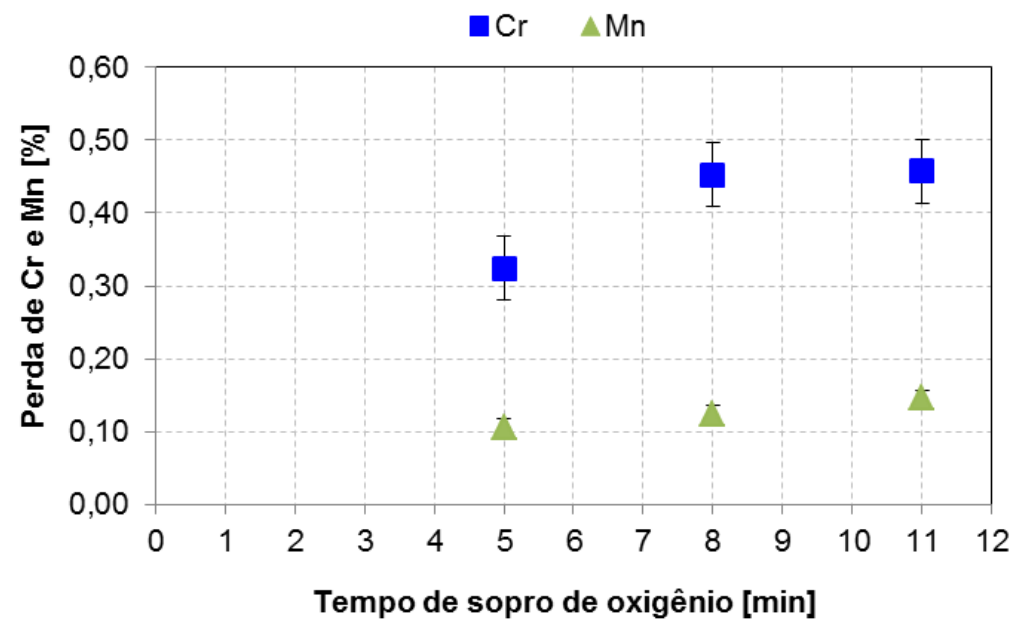

Figura 12: Influência do tempo de sopro na perda dos elementos de liga (valor médio de 10 corridas).

Os efeitos do teor de carbono e da temperatura inicial do processo, antes do sopro de oxigênio, podem ser vistos nas Figuras 13 (a) e (b). A perda de carbono é muito mais influenciada pelo tempo de sopro de $\mathrm{O}_{2}$ do que os valores iniciais de carbono e temperatura. De fato, a temperatura inicial tem influência somente nos instantes iniciais até a estabilização da pressão.

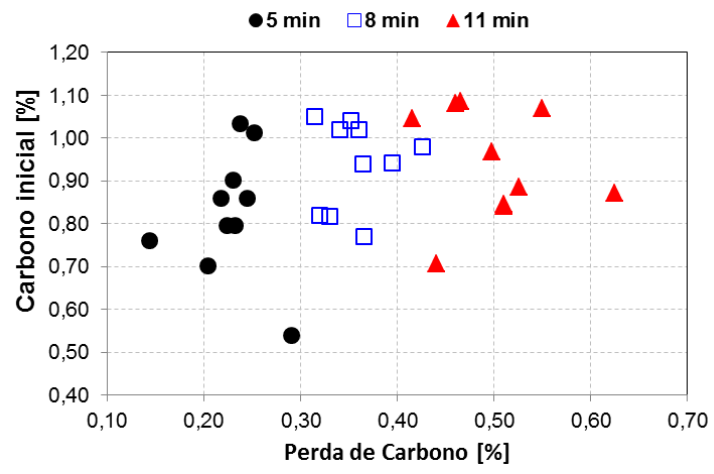

(a)

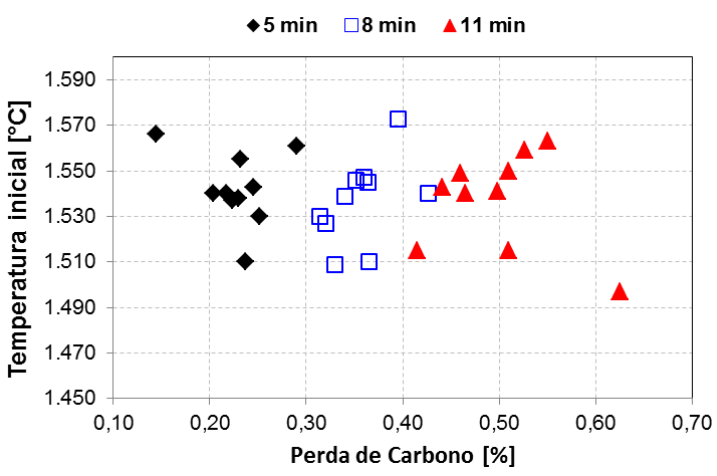

(b)

Figura 13: Perda de carbono em função (a) do carbono inicial e (b) da temperatura inicial para os diferentes tempos de sopro de oxigênio. 


\subsection{Cinética da Descarburação.}

De posse dos dados experimentais relativos ao processo de descarburação no estágio inicial de sopro de oxigênio, onde temos o carbono inicial $\left(\mathrm{C}_{\mathrm{i}}\right)$, assim como a utilização das Equações 4-8, foi possível então calcular o teor de carbono num dado tempo $\left(\mathrm{C}_{\mathrm{t}}\right)$.

$$
k_{R}=-\frac{\rho}{W} \cdot k \cdot A
$$

Onde $\mathrm{k}_{\mathrm{R}}=$ Constante da reação.

$$
\begin{array}{r}
\frac{d \% C}{d t}=k_{R} \cdot\left[\% C_{i}-\% C_{t}\right] \\
\int \frac{d \% C}{\left[\% C_{i}-\% C_{t}\right]}=\int k_{R} \cdot d t \\
-\ln \left[\% C_{i}-\% C_{t}\right]=k_{R} \cdot t+C \\
\ln \left[\% C_{i}-\% C_{t}\right]=0,1361 \cdot t-2,15
\end{array}
$$

Onde $\mathrm{t}=$ tempo de sopro de oxigênio expresso em $\mathrm{min}$.

A Figura 14 mostra a comparação dos dados reais obtidos nos testes com os valores calculados utilizando-se a equação 8 . Observa-se que existe boa correlação com uma variação de $\pm 0,1 \% \mathrm{C}$. Esta equação será utilizada futuramente para determinar o instante no qual a pressão total do sistema poderá ser reduzida.

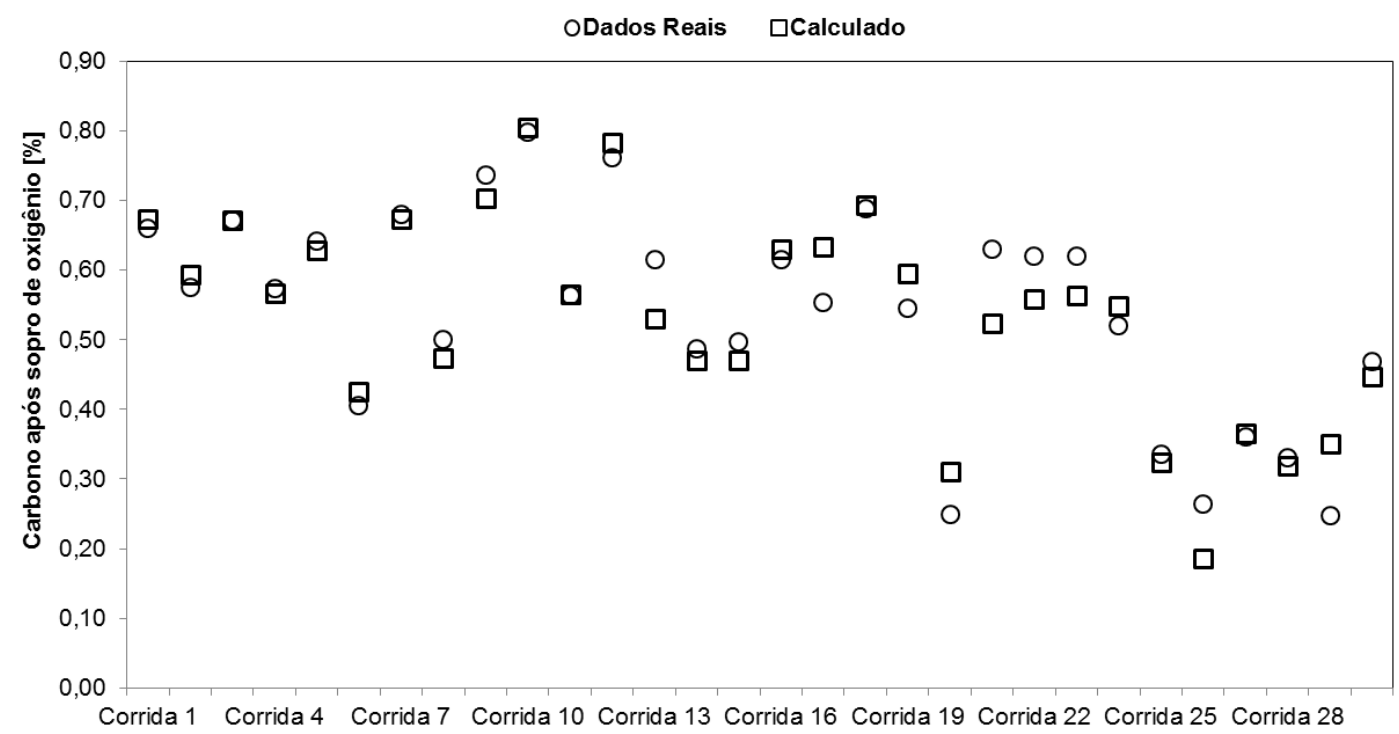

Figura 14: Dados reais da concentração de carbono após o sopro de oxigênio em comparação com os valores calculados utilizando a Equação 8. 


\section{CONCLUSÃO}

A perda de carbono é proporcional ao tempo de sopro e não depende do carbono inicial e também da temperatura inicial do processo, ficando estas variáveis implícitas no primeiro período de sopro, onde a pressão total do sistema oscila em torno de 600 mbar. Foi comprovado que é possível utilizar a equação cinética proposta e determinar o momento em que a pressão do sistema pode ser reduzida. Considerando um carbono inicial de $1 \%$ e objetivando um teor de $0,5 \%$ para a redução da pressão, o tempo de sopro após a estabilização inicial da pressão seria de $11 \mathrm{~min}$ sem que haja nenhum inconveniente de acordo com as condições operacionais, objetivando assim uma maior descarburação e consequentemente menor oxidação dos elementos de liga.

\section{REFERÊNCIAS}

1 International Stainless Steel Forum [página da internet]. Bruxelas: ISSF, 2015 [acesso em 01 ago. 2016]. Disponível em: http://www.worldstainless.org.

2 Associação Brasileira do Aço Inoxidável [página da internet]. São Paulo: ABINOX, 2016 [acesso em 10 ago. 2016]. Disponível em: http://www.abinox.org.br/aco-inox-estatisticas-anuais.php.

3 Hilty DC, Kaveney TF. Stainless Steel Melting. In: AIME. Taylor CR, Custer CC. Electric Furnace Steelmaking. Capitulo 13. Michigan: AIME. 1985: 143-160.

4 Patil BV, Chan AH, Choulet RJ. Refining of Stainless Steels. In: AISE. The Making, Shaping and Treating of Steel, Steelmaking and Refining Volume. Pittsburgh: AISE. 1998: 715-741.

5 Swinbourne DR, Kho TS, Langberg D, Blanpain B, Arnout S. Understanding stainless steelmaking through computational thermodynamics Part 2 - VOD. Mineral Processing and Extractive Metallurgy. 2010;119: 107-115.

6 Ding R, Blanpain B, Jones PT, Wollants P. Modeling of the Vacuum Oxygen Decarburization Refining Process. Metallurgical and Materials Transactions B. 2000; 31(B): 197-206.

7 Schöler HD, Maas H. Operation Technique and Plant Design of the VAD/VOD process. Metallurgical Plant and Tecnology. 1985; 6: 36-45.

8 Teeuwsen A. Vacuum oxygen decarburization (VOD) of stainless steel. Vakuum in Forschung und Praxis. 2014; 26: 32-37.

9 Silva ALVC, Mei PR. Aços e Ligas Especiais, 2. ed. São Paulo: Editora Edgard Blücher Ltda; 2006.

10 Turkdogan ET, Fruehan RJ. Fundamentals of Iron and Steelmaking. In: AISE. The Making, Shaping and Treating of Steel, Steelmaking and Refining Volume. Pittsburgh: AISE. 1998: 136-158. 
11 Meyer W. Optimisation Criteria for High Chromium Steel Production Lines. Siemens VAl's Iron and Steelmaking Conference Proceedings, Linz, October 911, Austria. 2006; 1-9.

12 Krishnakumar k, Ballal NB, Sinha PK, Sardar MK, Jha KN. Water Model Experiments on Mixing Phenomena in a VOD Ladle. ISIJ International. 1999; 39 (5): 419-425.

13 Bingel CJ, Griffith CB. Production of Low Carbon Steel by the VOD Process. Electric Furnace Conference Proceedings. 1972; 30: 51-54.

14 Cotchen JK, Eichert T, Tembergen D, Triplat J. Operating principle and plant design of the VD/VOD facility at Thyssen Krupp Acciai Speciali Terni for the production of superferritic stainless steel. Iron and Steel Technology. 2010; 7: 45-53.

15 Ribeiro DB. Refino Secundário dos Aços, Apostila do curso. São Paulo: Associação Brasileira de Metalurgia e Materiais; 2014. 